\title{
Quantification of the detailed cardiac left ventricular trabecular morphogenesis in the mouse embryo
}

\author{
Bruno Paun ${ }^{\mathrm{a}, *}$, Bart Bijnens ${ }^{\mathrm{a}, \mathrm{b}, \mathrm{c}}$, Andrew C. Cook ${ }^{\mathrm{d}}$, Timothy J. Mohun ${ }^{\mathrm{e}}$, \\ Constantine Butakoff ${ }^{a}$ \\ ${ }^{a}$ Universitat Pompeu Fabra, Department of Information and Communication \\ Technologies, c. Roc Boronat 138, 08018 Barcelona, Spain \\ ${ }^{b}$ ICREA, Passeig Lluís Companys 23, 08010 Barcelona, Spain \\ ${ }^{c}$ KU Leuven, Oude Markt 13, 3000 Leuven, Belgium \\ ${ }^{d}$ UCL Institute of Cardiovascular Science, University College London, Gower Street, \\ London, UK \\ ${ }^{e}$ The Francis Crick Institute, 1 Midland Road, London, UK
}

\begin{abstract}
During embryogenesis, a mammalian heart develops from a simple tubular shape into a complex 4-chamber organ, going through four distinct phases: early primitive tubular heart, emergence of trabeculations, trabecular remodeling and development of the compact myocardium. In this paper we propose a framework for standardized and subject-independent 3D regional myocardial complexity analysis, applied to analysis of the developmentevolution of the mouse left ventricle. We propose a standardized subdivision of the myocardium into 3D overlapping regions (in our case 361) and a novel visualization of myocardial complexity, whereupon we: 1) extend the fractal dimension, commonly applied to image slices, to 3D and 2) use volume occupied by the trabeculations in each region together with their surface area, in order to quantify myocardial complexity. The latter provides an intuitive characterization of the complexity, given that compact myocardium will tend to occupy a larger volume with little surface area while high surface area with low volume will correspond to highly trabeculated areas.

Using 50 mouse embryo images at 5 different gestational ages (10 subjects per gestational age), we demonstrate how the proposed representation
\end{abstract}

\footnotetext{
${ }^{*}$ Corresponding author. E-mail: bruno.paun@upf.edu. Postal address: Universitat Pompeu Fabra, Department of Information and Communication Technologies, c. Tànger 122 - 140, 08018 Barcelona, Spain
} 
and complexity measures describe the developmentevolution of LV myocardial complexity. The mouse embryo data was acquired using high resolution episcopic microscopy. The complexity analysis per region was carried out using: 3D fractal dimension, myocardial volume, myocardial surface area and ratio between the two. The analysis of gestational ages was performed on embryos of 14.5, 15.5, 16.5, 17.5 and 18.5 embryonic days, and demonstrated that the regional complexity of the trabeculations increases longitudinally from the base to the apex, with a maximum around the middle. The overall complexity decreases with gestational age, being most complex at 14.5. Circumferentially, at ages 14.5, 15.5 and 16.5, the trabeculations show similar complexity everywhere except for the anteroseptal and inferolateral area of the wall, where it is smaller. At 17.5 days, the regions of high complexity become more localized towards the inferoseptal and anterolateral parts of the wall. At 18.5 days, the high complexity area exhibits further localization at the inferoseptal and anterior part of the wall.

Keywords: 3D fractal analysis, cardiac trabeculations, high resolution episcopic microscopy, cardiac embryology, cardiac morphogenesis

\section{Introduction}

The development of the myocardium appears to be similar in many vertebrates. In particular, before the intramural cardiac vessels appear, the ventricular walls consist mainly of trabeculations. Those early trabeculations increase myocardial surface area and serve to increase myocardial oxygenation and nutrient delivery by diffusion, while sufficient tissue volume builds up for myocardium development at the later stages. However, the decrease in trabecular complexity does not lead to a disappearance of the trabeculations. A number of animal studies suggest that smooth ventricles lead to severe heart failure and elevated embryonic lethality (Captur et al., 2015). On the other hand, it was observed that excessive trabeculations can lead to heart failure, atrial and ventricular arrhythmias and thromboembolic events (stroke) (Ritter et al., 1997; Oechslin et al., 2000; Kovacevic-Preradovic et al., 2008; Penela et al., 2013; Oechslin and Jenni, 2011). Trabeculated myocardium can be seen in normal hearts and can be frequently seen at younger ages, diminishing with age, with some gender differences (Dawson et al., 2010). A high proportion of young athletes, especially of African/Afro-Caribbean origin, also exhibit an increased amount of trabeculations (Gati et al., 2013). 
One of the animal models to investigate the developing heart and related pathologies is the mouse embryo, where genetic alteration have been found to affect normal cardiac development, leading to either decreased or increased trabeculations. The trabeculae first appear at gestational age (GA) of about 9.0 - 9.5 embryonic days, at the end of cardiac looping. By GA14.5 ventricular septation is complete and a dense trabecular meshwork is established. At this moment, trabecular meshwork starts to become less complex and intertrabecular spaces seem to transform into the vessels (Captur et al., 2016). At the same time, papillary muscles, the moderator band, and effective arterial valves are starting to form.

Recently, a quantification of myocardial complexity of mice embryos was presented (Captur et al., 2016). The authors proposed to use fractal dimension (FD), measured on the myocardial contours in 2D short axis and 2-chamber MRI image stacks. The authors demonstrated that trabecular profiles increase from base towards the mid/apical region and decrease towards the apex. They also demonstrated that during later development, from the establishment of the interventricular septum at GA14.5 to GA18.5, trabecular complexity reduces and its decline is not proportional to the increase in compact wall volume. Even though the 2D FD measurement correlates with the visual perception of complexity, it has several drawbacks when applied to non-linear fractal structures such as natural fractals. The first and the largest problem in FD calculation is a lack of consensus with respect to the choice of its parameters. For example, in the typically used box counting, one has to decide on the minimum and maximum block size, the step for the block shift, how to perform the block shifts as well as how many to do, and finally, how to fit a line to the box counts (Foroutan-Pour et al., 1999; Jelinek et al., 2006; Karperien and Jelinek, 2016). Any variation in these parameters can significantly affect the FD. Another problem with 2D FD for analysis of myocardium is that the calculated values do not represent the $3 \mathrm{D}$ structure, and 2D slice-by-slice analysis inherently restricts the applicability of a larger range of methods.

In this paper, we propose a framework for 3D myocardial complexity assessment in terms of 3D FD and myocardial surface area to volume ratio. Such ratio represents an important parameter in diffusion processes and is widely used in chemistry, physics, biology and physiology (Latour et al., 1993; Yuan et al., 2010). To asses the myocardial complexity in each region, we propose to use a moving window (encompassing the neighboring regions), calculating the volume of the myocardium within the window and 
the area of the myocardial surface in contact with the blood. The surface area is expected to increase with the amount of blood cavities within the myocardium, while the volume increases with the amount of tissue filling the region. Therefore, highly trabeculated areas will have high area and low volume measures, while compact tissue will have low area and high volume. In our experiments, we demonstrate that the regional ratio of myocardial surface area to volume increases with myocardial complexity.

The proposed methodology was used to study the developmentevolution of cardiac trabeculations in the left ventricle (LV) of mouse embryos across different gestational ages. The framework is based on the recently introduced myocardium reparameterization (Paun et al., 2017), where an individual shape-independent method for establishing point-to-point correspondence between the cardiac ventricles is proposed. Consequently, that method provides a way of subdividing the ventricles into $3 \mathrm{D}$ regions in a standardized way for inter-individual comparison of structural and shape properties as well as studying temporal developmentevolution. The myocardial complexity can then be estimated on a per region basis with regions of any desired size. The study was performed on the set of 50 mouse embryos, acquired at different gestational ages: from GA14.5 embryonic days, when the ventricular septation is complete and a dense trabecular meshwork is established within the ventricular cavities, through GA15.5, GA16.5, GA17.5, to GA18.5 embryonic days, during the simultaneous processes of decrease in trabecular complexity and continued growth of the compact myocardial layer. The myocardium was subdivided into 361 regions (98 slices in longitudinal direction (apexbase) and 40 in circumferential direction (septal-inferior-lateral-anterior) plus apex). In each of these regions we calculated the 3D FD, myocardial volume, myocardial surface area, and surface to volume ratio. We show how these measures represent the myocardial complexity for each gestational age and how they do evolve from one age to another, emphasizing the differences. Finally we discuss the advantages and disadvantages of each of the measures.

\section{Data}

All specimens were handled in compliance with the Guide for the Care and Use of Laboratory Animals published by the US National Institutes of Health and with the approval of the MRC National Institute of Medical Research Ethical Review Panel. Mouse (Mus musculus) embryos were obtained from NIMR:Parkes (a robust outbred strain maintained at the MRC National 


\begin{tabular}{ll}
\hline & Table of abbreviations \\
\hline FD & fractal dimension \\
HREM & high resolution episcopic microscopy \\
GA & gestational age \\
LV & left ventricle \\
LAX & long axis \\
SR & surface ratio \\
VR & volume ratio \\
\hline
\end{tabular}

Table 1: List of abbreviations used in the manuscript.

Institute of Medical Research). For approximate embryo staging, detection of a vaginal plug was taken as gestation day 0.5 (GA0.5).

In order to minimize retention of blood in embryo hearts, harvested embryos were first agitated in phosphate buffered saline (PBS) solution at $37^{\circ} \mathrm{C}$ containing heparin for approximately 15 minutes (min), while all umbilical vessels being repeatedly clipped to allow blood to be pumped out. Potassium chloride was then added (final $50 \mathrm{mM}$ ) to ensure that hearts arrested in diastole. Hearts (including attached lungs and thymus) were then isolated, washed briefly in fresh PBS and after removal of at least one lung lobe, samples were fixed for $30 \mathrm{~min}$ in fresh $4 \%$ paraformaldehyde at $4{ }^{\circ} \mathrm{C}$. To remove remaining blood within the heart chambers, samples were then washed in repeated changes of distilled water over 30-60 min at room temperature with constant agitation (roller). The resulting osmotic shock lysed any remaining blood within the heart chambers without any alteration to heart structure as assessed by histology. After overnight fixation in $4 \%$ paraformaldehyde $\left(4^{\circ} \mathrm{C}\right)$, hearts were dissected away from associated lung, thymus and pericardial tissue prior to dehydration and embedding in methacrylate resin (Mohun and Weninger, 2012). Samples were similarly positioned during embedding to ensure relatively reproducible base-to-apex sectioning during the high resolution episcopic microscopy (HREM) imaging process.

The prepared samples were then used for the HREM analysis as described in Weninger et al. (2006). Briefly, HREM uses block-face imaging to produce perfectly registered digital image stacks capturing the 3D architecture of the embryonic heart at high resolution. Resulting datasets comprise 1000-2000 digital, short-axis images, produced by repeated removal of $2 \mu m$ (GA14.5 GA16.5) or $3 \mu m$ (GA17.5, GA18.5) sections (base-to-apex direction). The volumetric visualizations of the datasets from each GA can be seen in Fig. 1. 


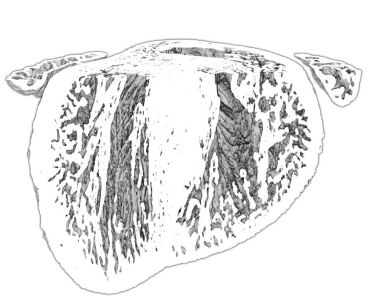

GA 14.5
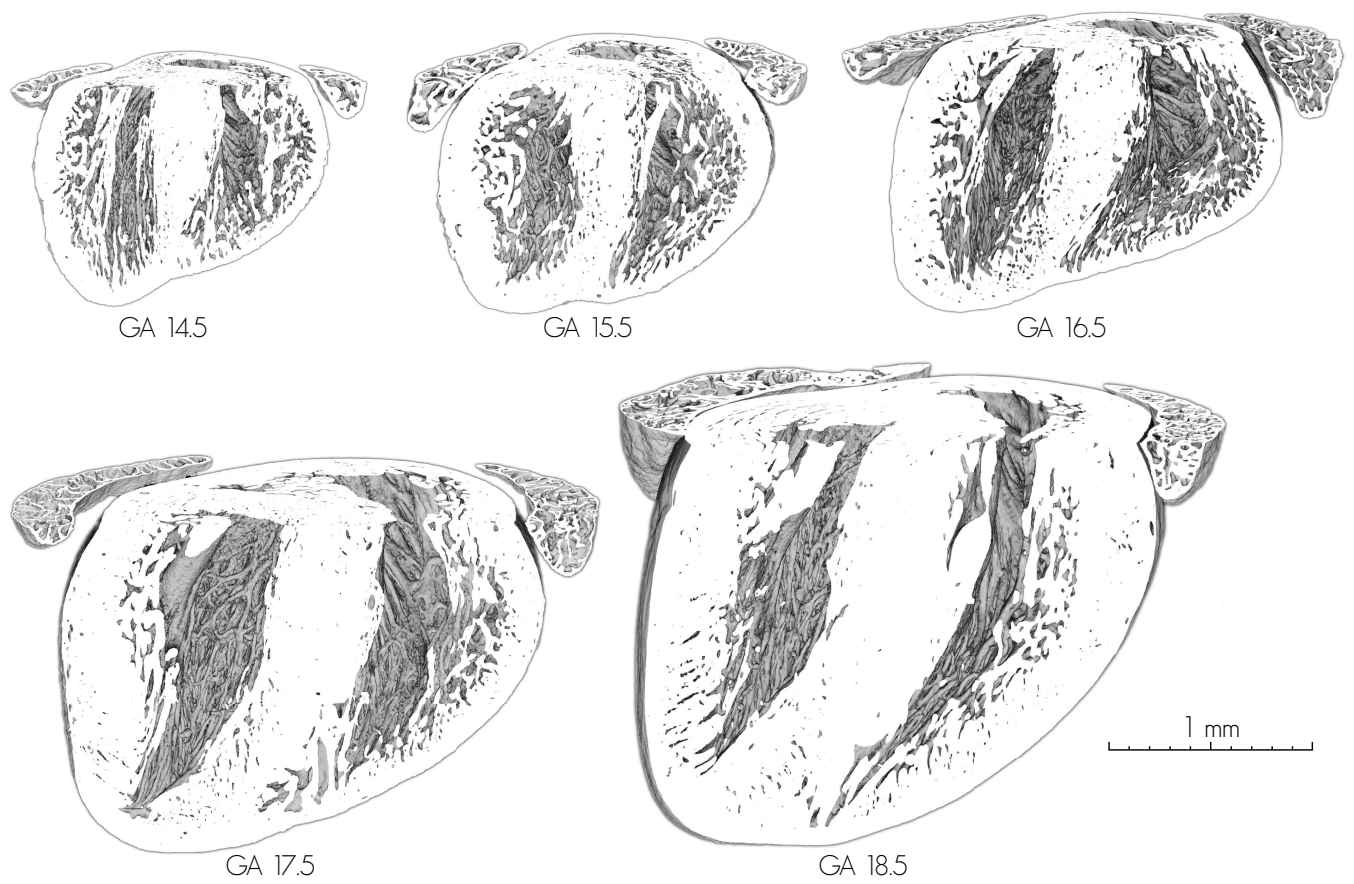

Figure 1: Volumetric visualization of the datasets for each GA. 


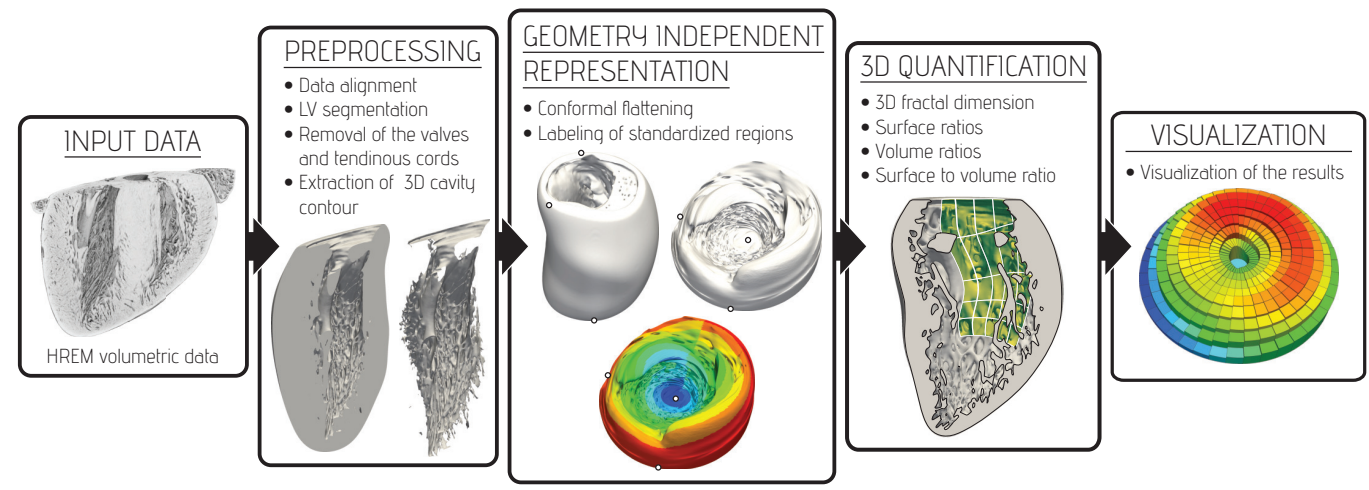

Figure 2: Pipeline for 3D quantification of myocardial complexity. The colors of the unfolded mesh under geometry independent representation represent the labels of 361 regions, while the dots depicted in all three figures represent three defined landmarks. The colors of the figure under visualization step represent myocardial volume values for each of 361 regions of an arbitrary dataset. The colors in these figures are used for illustrative purposes and are not representative of the final analysis results.

\section{Methods}

\subsection{Overview}

In Fig. 2, we depict an overview of the proposed framework. The input HREM datasets are first preprocessed, aligning them to have approximately the same orientation, and to segment the LV myocardium. We manually removed the valves and the tendinous chords, and from the cleaned segmentation, extracted the interface between the blood pool and the tissue. The cleaned segmentation and the interface images are then mapped to the standardized representation and subdivided into regions.

The tissue complexity analysis was performed on 50 subjects, 10 subjects per GA, ranging from 14.5 to 18.5 embryonic days. For each GA, mean and standard deviation of the region complexity values were calculated and plotted on continuous bulls-eye plots. Two metrics were used:

- Fractal dimension, performed on the interface between the trabeculations and the blood pool.

- Regional myocardial volume and surface area, performed on the myocardial image masks. 


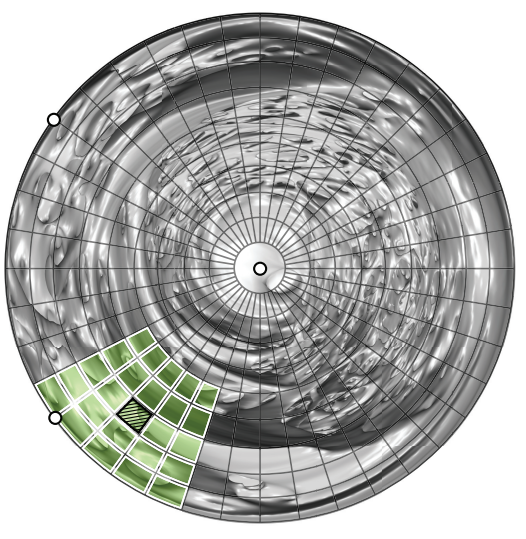

a)

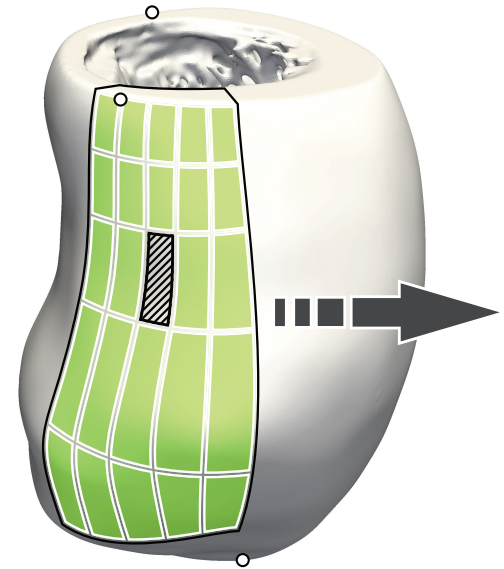

b)

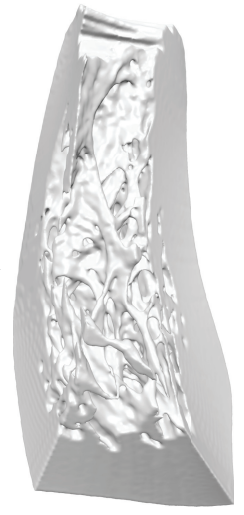

c)

Figure 3: Visualization of one of the datatset's region and its neighborhood used in the analysis. a) Unfolded dataset divided into 361 regions. The black shaded element represents the region for which we calculate the corresponding metrics, while the green elements represent its $5 \times 5$ neighborhood region used in calculations. b) The same element and its neighborhood region displayed on the original non-flattened dataset on which corresponding metrics are calculated. c) Extracted region and its $5 \times 5$ neighborhood region viewed from the inside of the cavity. The three dots depicted in figures a) and b) represent anterior septal, posterior septal and apical landmarks, defined to remove orientation ambiguity in the normalized domain. The process of obtaining those landmarks is explained in detail in Paun et al. (2017).

To produce the plots of complexity, the analysis was performed on the neighborhood of each region by employing a $5 \times 5$ moving window (Fig. 3). The values on the boundaries were calculated by zero padding. The neighborhood of the apical region was defined as the apex and the adjacent apical ring regions. All complexity metrics were calculated on the original datasets.

\subsection{Data preprocessing}

The proposed framework for 3D assessment of myocardial complexity takes as an input aligned volumetric image segmentations of LV myocardium with all its detailed anatomy present within the blood pool (excluding valve and tendinous chords). The alignment was corrected by matching the heart's LV long axis (LAX) (defined by the centroid of the mitral annulus and the LV apex) with the positive $\mathrm{z}$ direction of the image. 
The whole heart segmentations of aligned datasets were first obtained in Fiji (an image analysis software (Schindelin et al., 2012)) by using automatic global thresholding based on Rènyi's entropy algorithm, as it has been shown that it outperforms other entropy based thresholding methods (Sahoo et al., 1997). Subsequently, the whole hearts segmentations were imported to the Seg3D image processing software (CIBC, 2015) where their LVs were segmented similarly to the procedure described in (Paun et al., 2017). The imported whole heart segmentations were first clipped at the level of the Mitral valve annulus to remove the atria and great vessels. From those images, we extracted the LV blood pool mask and manually delineated and extracted the LV epicardial mask. The mitral valve and tendinous cords were manually delineated and removed from the blood pool masks. The myocardial mask, with all the detailed anatomy, was obtained as the difference of the epicardial and the cleaned blood pool mask. These are used to subdivide the myocardium into regions and perform assessment of myocardial complexity.

The proposed 3D fractal analysis is performed on a one voxel thick 3D contour, representing the interface between the myocardium and the blood pool, obtained using the binary contour filter (ITK (Yoo et al., 2002)) on the cleaned blood pool mask.

\subsection{Geometry independent representation of a ventricle}

The method of Paun et al. (2017) allows establishing correspondence between different objects having an overall shape of a thick hemispherical shell. To do so, apart from the object itself (represented by either a surface or volumetric mesh), we also require an enclosing surface (represented by a triangulated mesh), that contains the whole object in its interior. The mapping is then calculated in 2 steps. First, we map the enclosing surface to a suitable planar domain. To impose a consistent orientation, we specify 3 landmarks on the enclosing surface and perform the conformal or quasiconformal flattening to the planar domain. Secondly, we map our object to the volumetric domain defined by the planar domain (usually a cylinder or a prism). The planar domain is transformed as explained in Paun et al. (2017) to compensate for increasing triangle density towards the disk's center. In this volumetric domain, we subdivide the base (the disk) into 361 regions by introducing 98 rings of equal thickness (corresponding to the apex-to-base slices in the LV), each split equally into 40 circumferential (septal-lateralseptal) regions. The apical region is not subdivided. The subdivision can be seen in Fig. 3. The detailed sensitivity analysis of our representation to the 
variations in the landmark placement, as the analysis of distortion caused by mapping, can be found in Paun et al. (2017).

\subsection{Fractal analysis}

The 3D fractal analysis presented in this study was performed using an in-house implementation of the cube-counting method (3D equivalent of the box-counting method) similarly to Goñi et al. (2013), where the estimated fractal measures were obtained by the box counting method of Russell et al. (1980), suitable for objects with and without self-similarity.

The cube(box) counting method is performed by superimposing the 3D image of the object under study with isotropic grids made of regular cubes of size $\epsilon$. In the image, the object is represented by nonzero voxel values (with zero being the background).

We first find the smallest region enclosing the object (its bounding box) and randomly select the grid origin from all possible voxel locations within the region. For each grid of cube size $\epsilon$, the number of cubes necessary to fully cover the object, $N(\epsilon)$, is recorded. Let $N_{i}(\epsilon)$ represent the number of non-zero voxels contained in the $i$-th non-empty cube of size $\epsilon$, where $i \in[1, N(\epsilon)]$. The total number of non-zero voxels (voxels of the object) can be obtained from:

$$
N_{\text {all }}=\sum_{i=1}^{N(\epsilon)} N_{i}(\epsilon)
$$

and the probability of finding a non-zero voxel (voxel of the object) within the $i$-th non-empty cube of size $\epsilon$ is equal to:

$$
p_{i}=\frac{N_{i}(\epsilon)}{N_{\text {all }}} .
$$

Those measurements were then used to calculate the generalized dimension spectrum (Hentschel and Procaccia, 1983), where generalized (Rènyi) dimensions $D_{q}$ of order $q$ are defined by:

$$
D_{q}=\lim _{\epsilon \rightarrow 0} \frac{1}{1-q} \frac{\log \sum_{i=1}^{N(\epsilon)} p_{i}^{q}}{\log \frac{1}{\epsilon}}, q=0,1,2, \ldots
$$

where $D_{q}$ is a non-increasing function of $q$,

$$
D_{q_{1}} \geq D_{q_{2}} \quad \text { if } \quad q_{1}<q_{2} .
$$


$D_{q}$ for $q>0$ will tend to be more accurate, as the higher order correlations are taken into account as $q$ increases. As can be seen from equation 3, for $q=0$, the $D_{0}$ dimension does not distinguish boxes with high density of voxels from those containing just a single voxel. More precise dimension measurements can be obtained from $D_{1}$ and $D_{2}$, which take into account inhomogeneities or correlations of elements of the object.

We calculated the generalized dimensions for $q=0,1$ and 2 which corresponds to the box counting dimension $\left(D_{0}\right)$, the information dimension $\left(D_{1}\right)$ and the correlation dimension $\left(D_{2}\right)$ respectively, where:

$$
\begin{gathered}
D_{0}=\lim _{\epsilon \rightarrow 0} \frac{\log N(\epsilon)}{\log \frac{1}{\epsilon}} \\
D_{1}=\lim _{\epsilon \rightarrow 0} \frac{\sum_{i=1}^{N(\epsilon)} p_{i} \log p_{i}}{\log \epsilon} \\
D_{2}=\lim _{\epsilon \rightarrow 0} \frac{\log \sum_{i=1}^{N(\epsilon)} p_{i}^{2}}{\log \epsilon}
\end{gathered}
$$

As the limit $\lim _{\epsilon \rightarrow 0}$ is not feasible in practical applications, $N(\epsilon)$ and $N_{i}(\epsilon)$ are measured for a certain range of $\epsilon$ values (Hentschel and Procaccia, 1983).

In our implementation, the grid cube size $\epsilon$ was linearly increasing by 1 voxel. The number of non-empty cubes decreases exponentially with the increase of the grid resolution $\epsilon$, where the exponent represents the corresponding fractal dimension depending on the order of correlations between image voxels taken into account. $D_{0}$ is estimated from the slope obtained by linear regression from the $\log -\log$ plot of $\log N(\epsilon)$ versus $\log 1 / \epsilon$. Similarly, $D_{1}$ and $D_{2}$ are obtained from the $\log$-log plots of $\sum_{i=1}^{N(\epsilon)} p_{i} \log p_{i}$ versus $\log \epsilon$ and $\log \sum_{i=1}^{N(\epsilon)} p_{i}^{2}$ respectively.

Due to inter-object difference in geometry, grids of cube size $\epsilon$ do not represent the same spatial resolution between objects (Goñi et al., 2013). Moreover, it is known that using grids of too small and too big cube sizes will lead to erroneous fractal dimension estimates (Foroutan-Pour et al., 1999). To take into account the varying geometry size and possible variation in image resolution, the range of $\epsilon$ values was taken from $2 \%$ to $25 \%$ of the smallest dimension of the object bounding-box. The maximum grid cube size value of $25 \%$ was chosen as in the work of Foroutan-Pour et al. (1999) and Zhao et al. (2016), while the minimum cube size value was chosen as in 
Captur et al. (2016) and Zhao et al. (2016). An example of the calculations can be seen in Fig. 4, 3rd column.

In all our experiments, the values of $D_{0}, D_{1}$, and $D_{2}$ were similar, however the values corresponding to lower $q$ tended to be noisier and sometimes underestimating the real complexity. Since the higher $q$ tends to be more accurate, in all the following sections, as a fractal dimension (FD), we report only the value of $D_{2}$.

\subsection{Volume and surface area calculations}

Another type of complexity analysis is performed by utilizing volume and surface area measures of the object regions and their ratios. Given the regions defined on each dataset, we calculate the volume, occupied by the cardiac tissue for each region of the object, and the volume of the region itself (containing cardiac tissue and blood pool). The ratio of these two volumes provides a percentage of how much volume does the cardiac tissue occupy within the region. This ratio increases with the increase of wall thickness and compactness of the myocardium.

In a similar way, we calculate the surface areas of the cardiac tissue and the enclosing region. Surface areas are calculated from triangular surface meshes obtained form the corresponding volumetric images. To minimize computational time, data processing and influence of meshing parameters, the surfaces were obtained by applying the Marching Cubes algorithm (Lorensen and Cline, 1987). The ratio of cardiac tissue surface area and region surface area increases as the myocardial surface becomes more complex and convoluted within the region. Examples of the calculations can be seen in Fig. 4, 4th and 5th columns.

\section{Results}

\subsection{Complexity analysis using regional fractal measures}

The mean and standard deviation of the regional FD was calculated for each GA. In the cube-counting method, we used 50 grid offsets and the size of the grid boxes was ranging from $2 \%$ to $25 \%$ of the region's bounding box. The increment of the grid cube size was 1 voxel. Fig. 5 shows the mean regional correlation dimensions $\left(D_{2}\right)$ and their corresponding coefficients of variation per GA, expressed as the percentage of the mean.

From Fig. 5, 2nd column, we can see that myocardial complexity globally increases as we move from the base towards mid/apical part of the heart and 


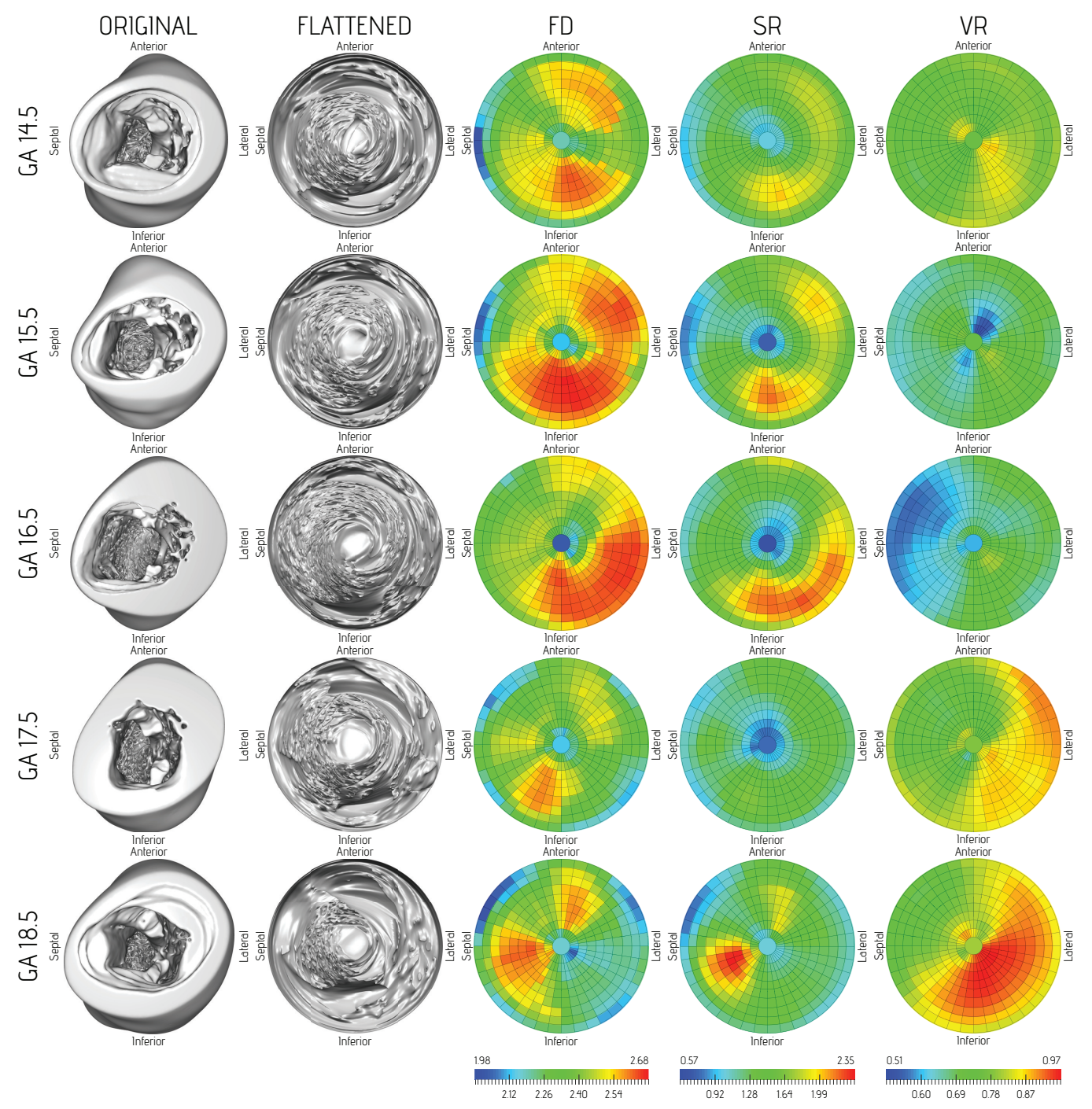

Figure 4: Sample datasets from each gestational age (GA) with the unfolding, FD $\left(D_{2}\right)$, surface ratio (SR) and volume ratio (VR). The datasets were clipped at the middle to give a better view at the interior of the chamber. 
then decreases towards the apex. That is especially pronounced at GA14.5, GA15.5 and GA16.5, where all mid and apical parts (except the anteroseptal, lateral and inferolateral wall) have similar trabecular complexity. The circumferential extent of the lower complexity regions increases from GA14.5 to GA16.5, while the lateral low complexity region, present at GA14.5, gradually shifts towards the inferolateral part of the wall. By GA17.5, the higher complexity is present in the mid and apical parts of the septal, inferior, and inferoseptal wall and the basal and mid parts of the anterior and anterolateral wall. The overall complexity globally decreases, which could be related to the simultaneous processes of decrease in trabecular complexity and the increase of the volume of the compact myocardial layer (Fig. 5, 3rd row, 1st column). At GA18.5, the most complex regions are narrowed to the mid and apical parts of the anterior, septal, inferior and inferoseptal wall. The regions of low complexity in the first and second basal ring, throughout all the GAs, are due to a smoother wall close to the outflow tract and the manually removed valve. As expected, myocardial complexity decreases with gestational age, which coincide with the visual inspection of the 3D data visualizations and is in agreement with results obtained by Captur et al. (2016).

The values of the coefficient of variation are more or less similar between the cases, while the areas of high variability are present in the apex of GA14.5 and apical, anterolateral regions of GA18.5.

Fig. 6 depicts plots of mean FD per GA. Nine different graphs in each plot represent FD values as we move circularly counterclockwise from the lateral wall towards the septum and back to the lateral wall. Each line represent a different radial location (ring) in the bull's eye plot, or longitudinal location in the apex-base direction. There are 10 graphs, with the apex being a straight black line, and 9 graphs representing 9 rings from the 1st apical ring (dark blue) to the 9th basal ring (dark red). The gray bands represent the average $95 \%$ confidence intervals over the population.

To show overall change of the mean FD in longitudinal direction, we averaged mean FD values per longitudinal regions and displayed how those values change with the GA. Fig. 7 shows ten different graphs representing mean FD values at different location along LAX for all GAs. The gray bands represent the average $95 \%$ confidence intervals over the population.

\subsection{Complexity analysis using regional occupied volume and surface area}

The overall occupied volume increases with gestational age, mainly due to myocardial wall thickening, which can be seen from Fig. 8 in the 2nd 


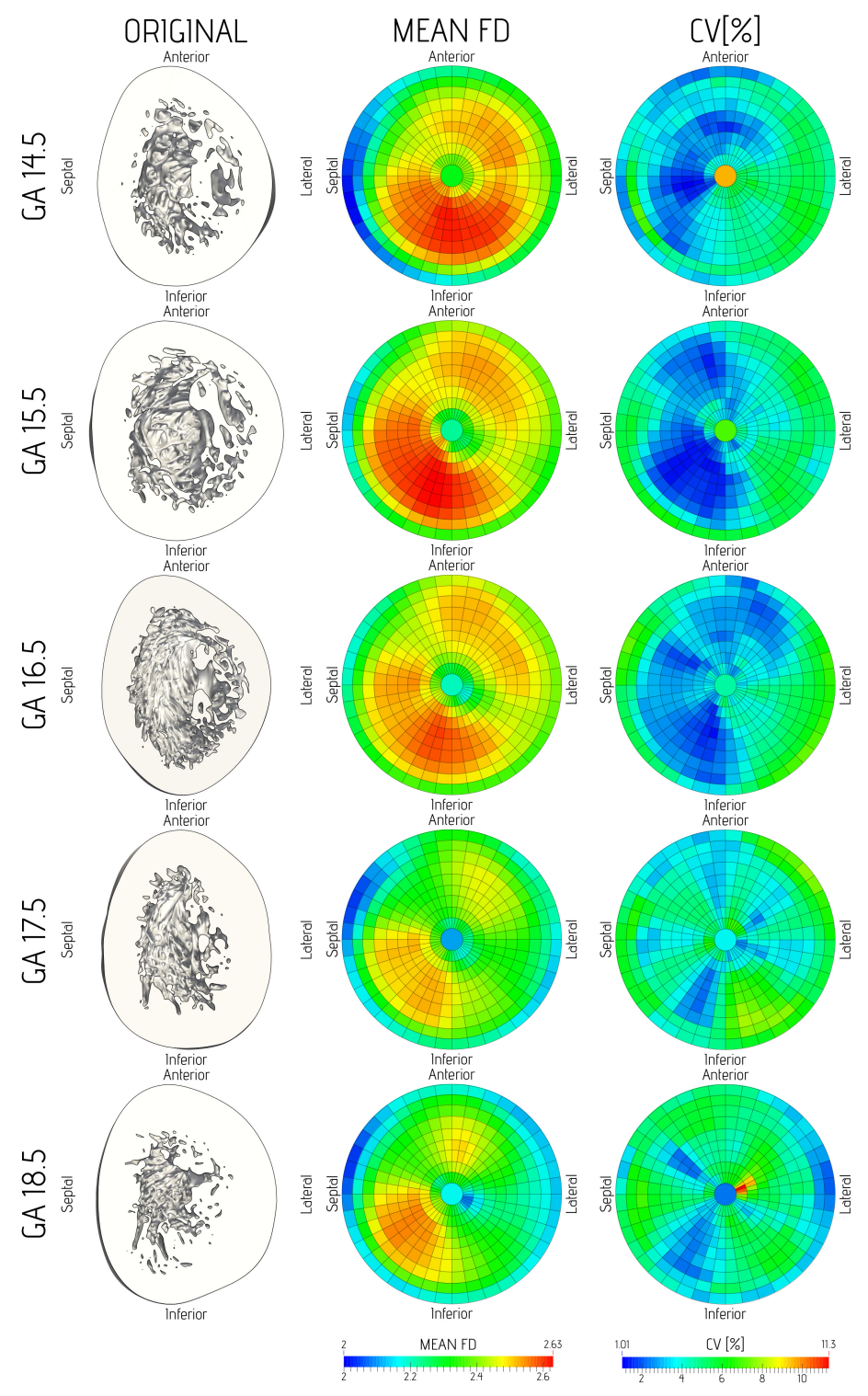

Figure 5: Continuous bull's eye plots representing the average regional fractal dimension $\left(D_{2}\right)$ per GA (2nd column) and the coefficient of variation of the regional fractal dimensions per GA (3rd column). The first column shows a sample dataset for each GA sliced at the middle. 

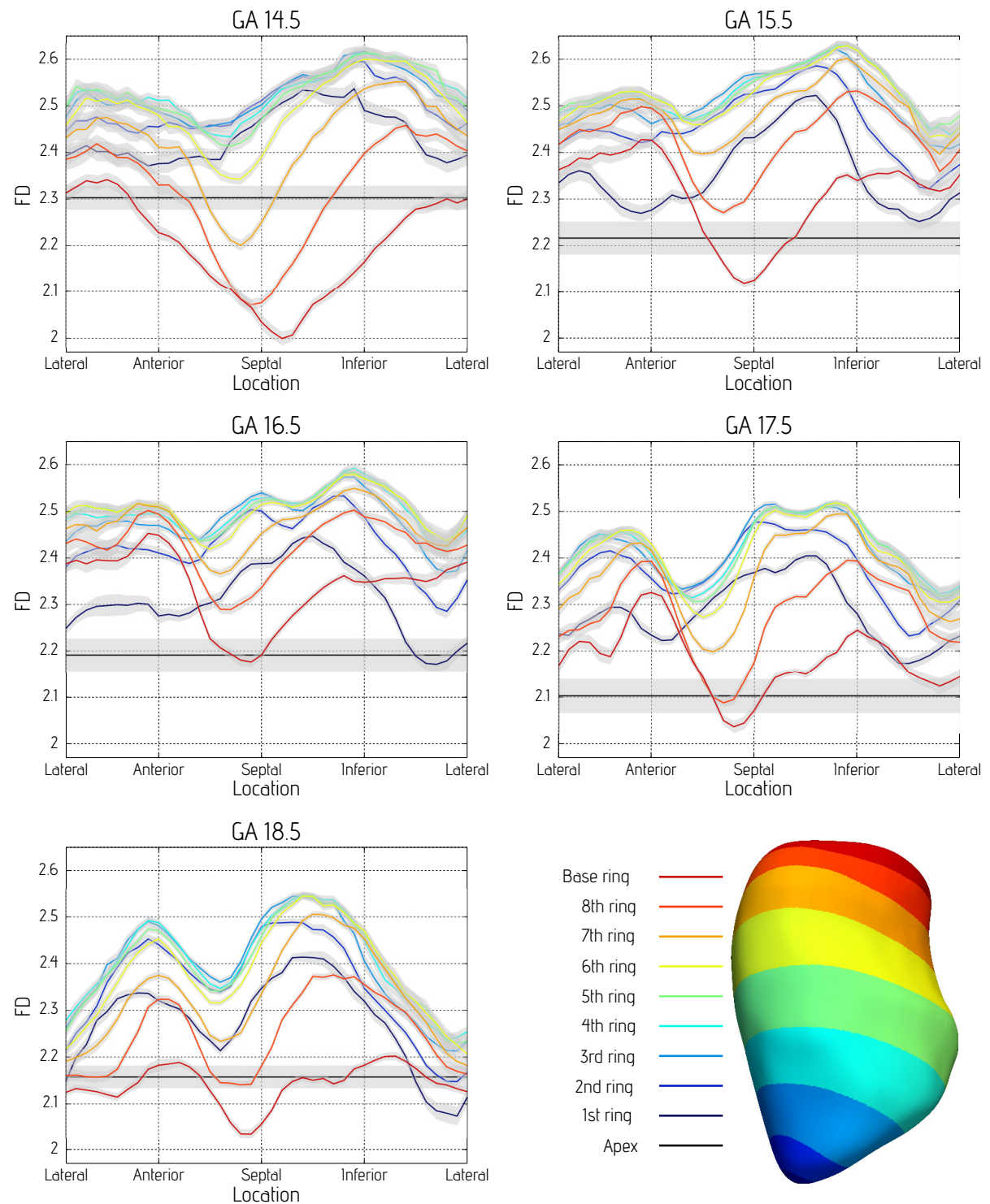

Figure 6: Plots of mean FD $\left(D_{2}\right)$ per GA. Data is grouped into 9 longitudinal regions (apex-base). The $\mathrm{x}$ axis represents FD values of the circular regions in the order: lateral anterior - septal - inferior - lateral wall. The gray bands represent average $95 \%$ confidence intervals over the population. 


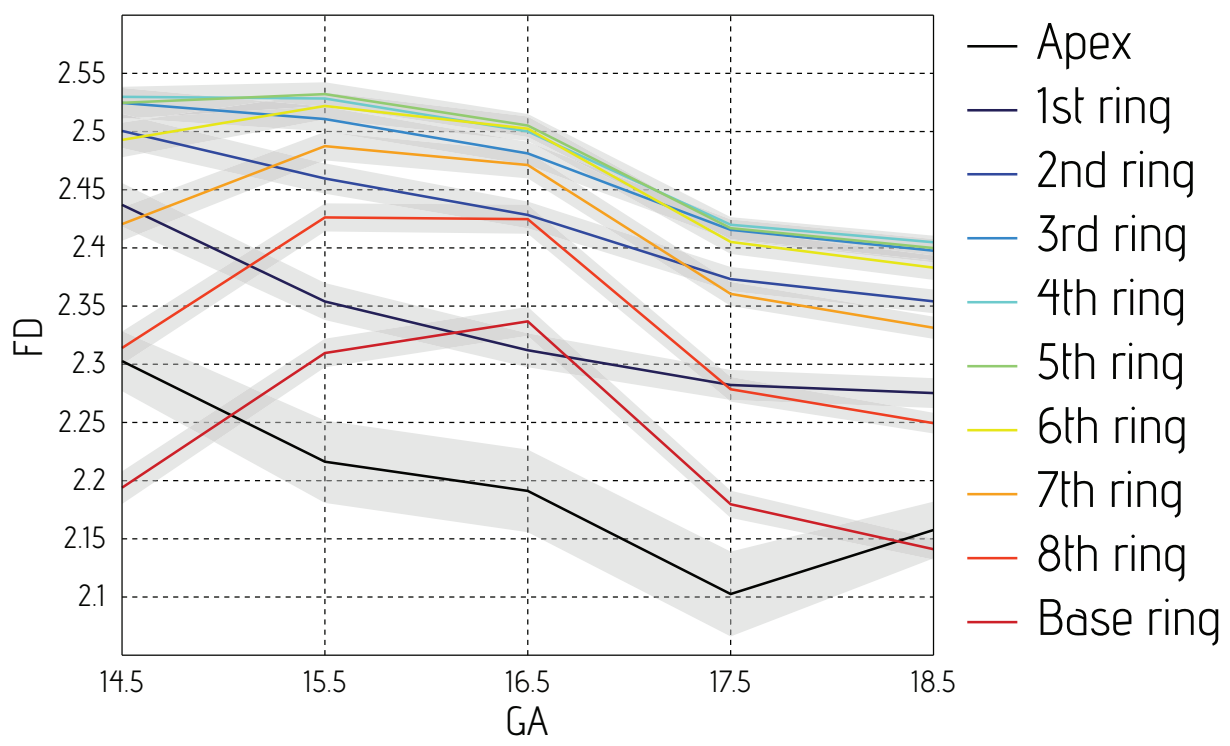

Figure 7: Plots of mean FD $\left(D_{2}\right)$ per longitudinal regions across different GAs. Mean FD values are reported for 9 longitudinal regions in LAX direction. The gray bands represent average $95 \%$ confidence intervals over the population.

column, representing the mean regional occupied volume. At GA14.5, the occupied volume is the highest in the apex, the basal, mid and apical parts of the inferior and inferolateral wall, the apical and mid parts of the lateral, anterolateral and anterior wall. At GA15.5, it increases everywhere, having the highest values at the mid and apical parts of the inferoseptal wall. By GA16.5 we see a significant decrease in the apex, the basal and mid anterior, anteroseptal and septal wall. A further overall increase can be seen by GA17.5, with highest values in the basal, mid and apical parts of the inferior, inferolateral and lateral wall and the basal parts of the anterolateral wall. At GA18.5, the overall occupied volume still increases, with the most pronounced increase in the basal, mid and apical parts of the inferior, inferolateral, lateral and anterolateral wall.

Fig. 8, 3rd column, represents the coefficient of variation of the regional occupied volumes within the population, expressed as the percentage of the mean. At GA14.5, the occupied volume between subjects varies the most in the apex, the apical regions of the inferior and anterolateral wall and the basal parts of inferoseptal wall. By GA15.5, the variation increases in the apical 


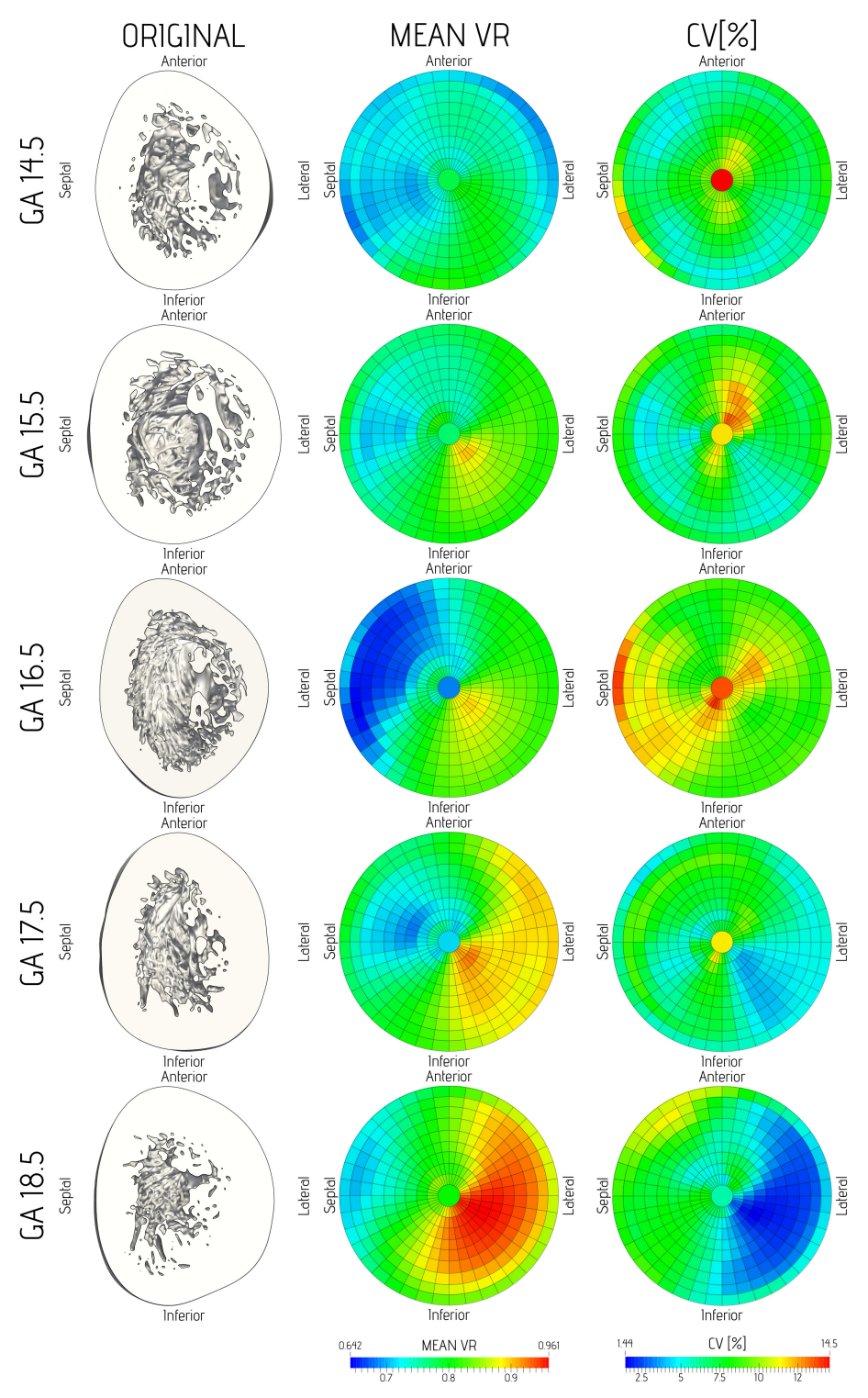

Figure 8: Continuous bull's-eye plots of average regional occupied volume per GA (2nd column) and coefficient of variation of regional occupied volumes per GA (3rd column). 
and mid parts of the anterior, anterolateral and lateral wall. At GA16.5 we observe an overall increase, where regions with the highest variability are located at the apex, the apical and mid parts of the anterolateral, lateral, inferior and inferoseptal wall, and the base and mid parts of the inferoseptal, septal and anteroseptal wall. At GA17.5, we observe an overall decrease with regions of low variability at the apical, mid and basal parts of the inferolateral wall. At GA18.5, a significant decrease of variation is observed in the whole inferior, inferolateral and lateral wall and the mid and basal part of the anterolateral wall. The regions of high variability in the basal parts throughout the GAs represent a segmentation artifact of the manual valve removal.

In Fig. 9, we show the mean and coefficient of variation of the regional surface area ratios. Higher values mean that the surface is more convoluted within the region. At GA14.5, the highest mean regional surface area ratios are present at the mid and apical parts of the inferior and inferolateral wall and the mid part of the lateral and anterolateral wall. At GA15.5, it also increases in the mid part of the anterior, septal, inferoseptal wall, while it decreases in the apex, the apical and mid parts of the inferior and inferolateral wall and the mid part of the lateral wall. At GA16.5 and GA17.5, we observe a further overall decrease, with the highest decrease in the apex and apical parts of the whole wall. Overall low surface ratio values, uniformly distributed over whole wall, are the results of decrease in trabecular complexity, making the endocardial surface less convoluted. By GA18.5, the value increases at the apex and is the highest at the apical and mid parts of the septal, inferoseptal and inferior wall, and the apical and mid parts of anterior wall.

The coefficient of variation of the regional surface area ratios at GA14.5 has uniformly low values everywhere. At GA15.5, it increases at the apical, mid and basal parts of the inferoseptal, anterolateral and anterior wall and at the apical and mid parts of septal and anteroseptal wall. By GA16.5, the variation in surface area ratios concentrates at the apex, the basal and mid parts of the inferolateral, lateral and anterolateral wall, the apical, mid and basal parts of the septal wall and the basal parts of the anteroseptal wall. At GA17.5, there is a propeller-like pattern, where the higher values are located in the mid and apical parts of the inferoseptal, lateral and anteroseptal wall. The peak values are located at the apical and mid part of the anteroseptal and lateral wall. Finally, at GA18.5, the pattern of moderate values is located at the apical and mid parts of the septal wall, the basal parts of anteroseptal 


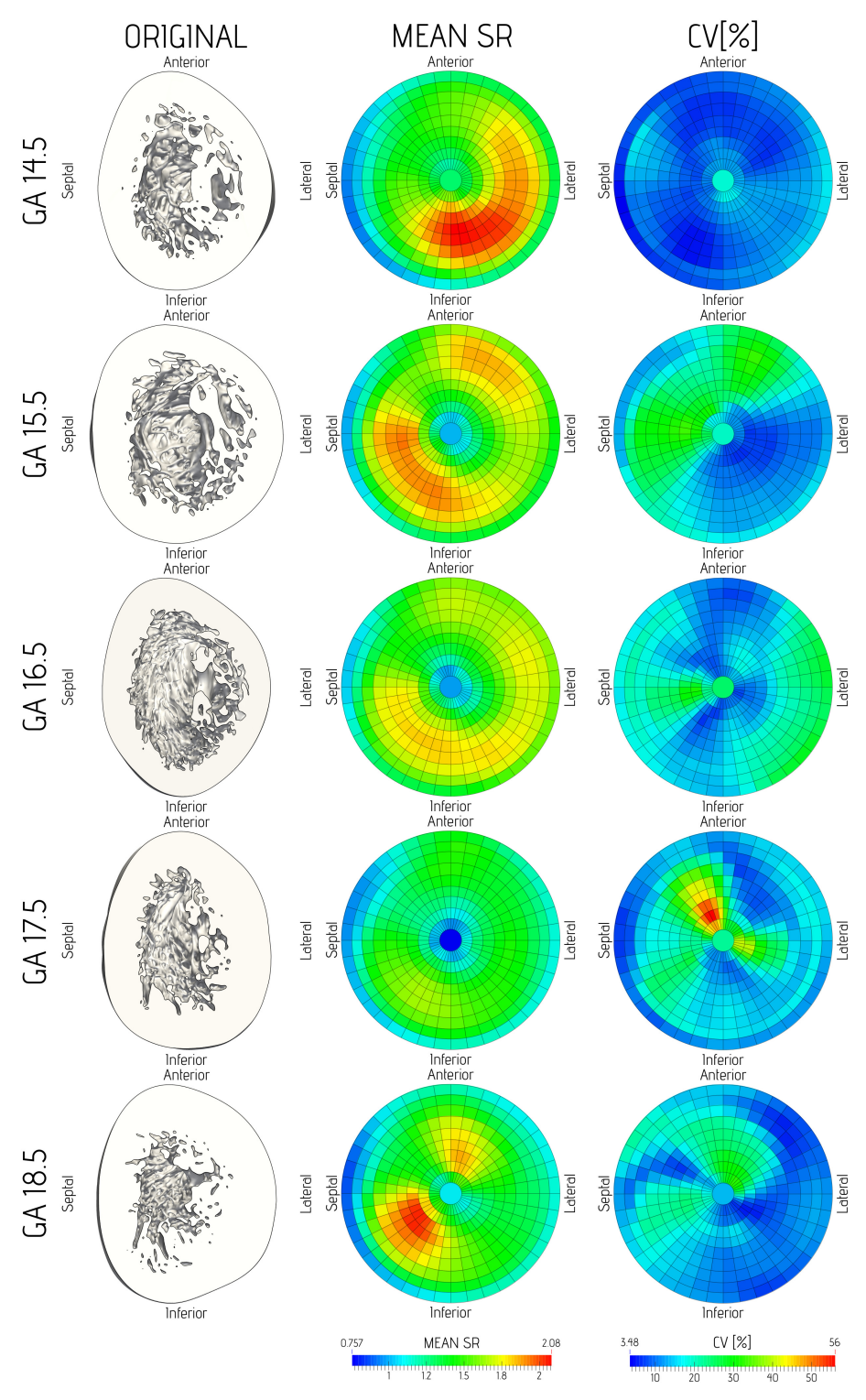

Figure 9: Continuous bull's-eye plots representing average regional surface area ratios per GA (2nd column) and coefficient of variation of regional surface area ratios per GA (3rd column). 
wall and the apical and mid parts of the anterior and anterolateral wall.

In Fig. 10, we show the mean and coefficient of variation of the normalized regional surface to volume ratios. The tissue surface areas and volumes were normalized by surface areas and volumes of the corresponding whole regions including tissue and cavity. Higher values indicate that the tissue surface is more convoluted within the region and occupy smaller volume. At GA14.5, the highest mean regional values are present at the mid and apical parts of the inferior and inferolateral wall and the mid part of the lateral and anterolateral wall. At GA15.5, its value increases in the mid part of the anterior, septal, inferoseptal wall, while it decreases in the apex, and all parts of the inferior, inferolateral and lateral wall. At GA16.5 and GA17.5, we observe a further overall decrease, with the highest decrease in the apex and the inferolateral and lateral wall. Overall low values are the results of decrease in endocardial surface complexity and increase of occupied volume. By GA18.5, the value increases at the apex and is the highest at the mid parts of the septal and inferoseptal wall, and the apical parts of anterior wall.

Fig. 11 shows a scatter plot of the myocardial volume versus surface area (not normalized by the region volume and area) for all 361 regions of the 5 GAs. For each GA, we fit a line passing through the origin. The correlation coefficients with $95 \%$ confidence intervals for each GA are the following: GA14.5: 0.956 [0.949,0.966], GA15.5: 0.956 [0.947,0.964], GA16.5: 0.967 [0.960,0.973], GA17.5: 0.9173 [0.899,0.932], GA18.5: 0.862 [0.833,0.886]. We can observe that the slope of the line increases with gestational age, correlating with the apparent increase of the volume of the compact myocardial layer and decrease in trabecular complexity with GA. Thus, if we look at surface to volume ratio, myocardial complexity is the highest at GA14.5 and decreases towards GA18.5. The highest increase in the slope of GA17.5 with respect to GA16.5 could be explained due to growth of the compact myocardial layer and likely nonlinear progression of complexity evolution, which we can observe in the bull's eye plots. In particular, we can see that at GA17.5, the overall occupied volume increased, while the overall surface area decreased considerably, thus leading to a higher jump in the slope of the GA17.5 regression line.

For all calculated values, namely fractal dimension (FD), surface to volume ratio $(\mathrm{S} 2 \mathrm{~V})$ and normalized surface to volume ratio (SR2VR) we performed statistical test between GAs. Fig. 12 depicts box plots of calculated values for all the regions of the datasets grouped by GA. We calculated two sided Wilcoxon rank sum test between GAs and obtained statistical signif- 


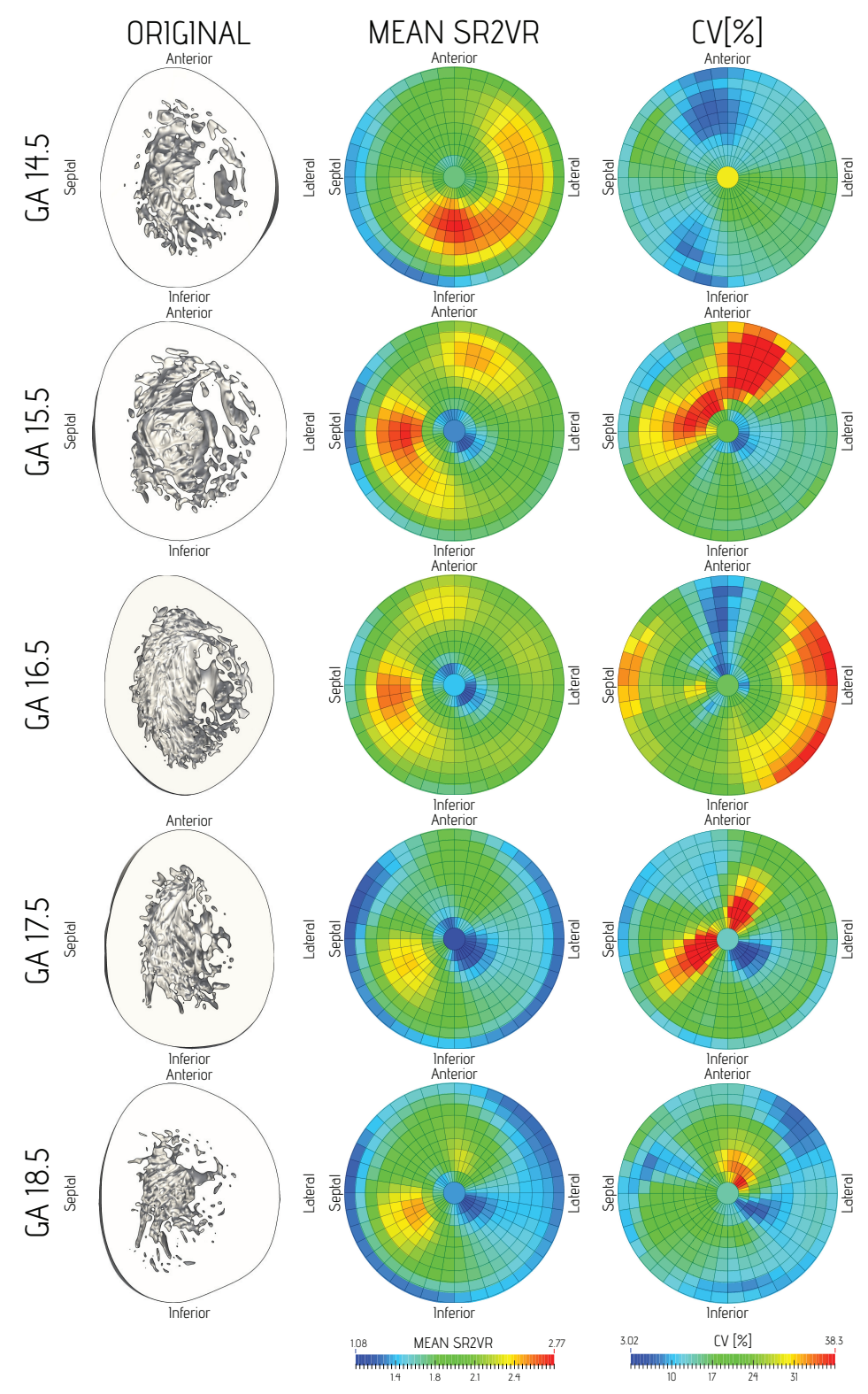

Figure 10: Continuous bull's-eye plots representing average regional normalized surface area to occupied volume ratios per GA (2nd column) and corresponding coefficients of variations per GA (3rd column). 


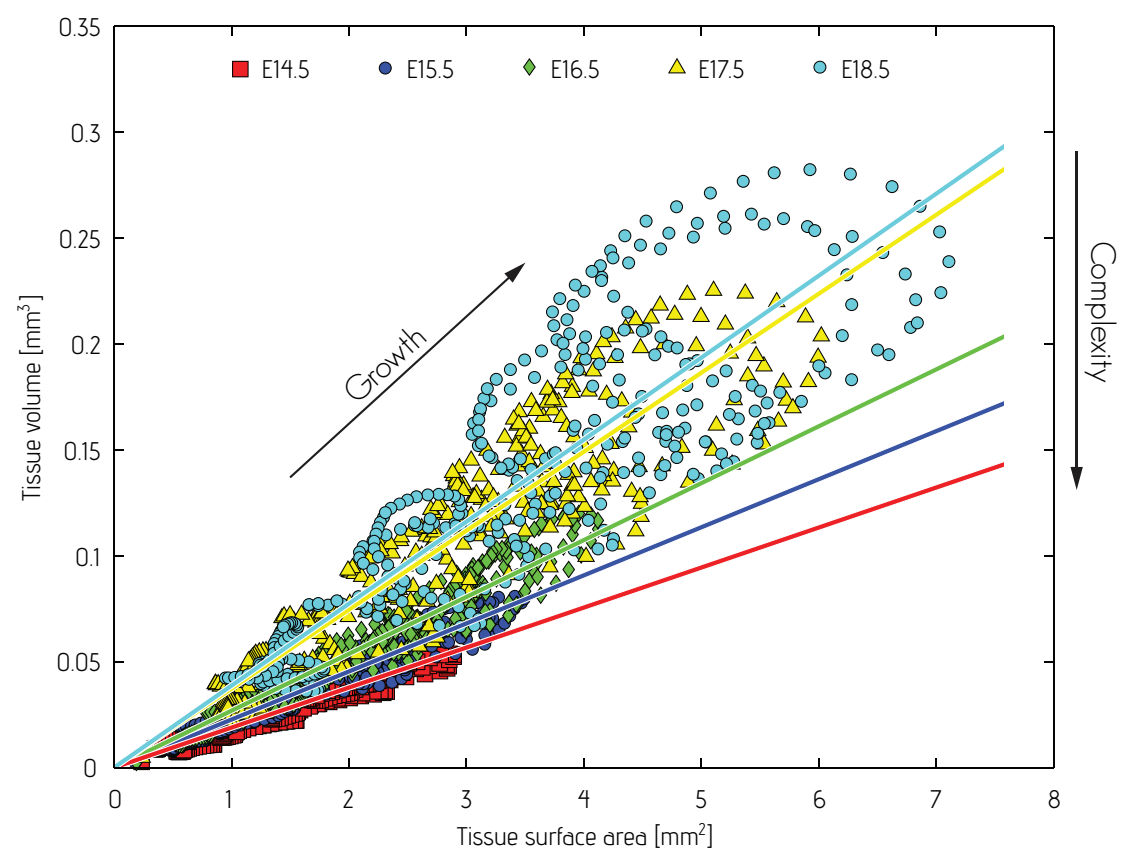

Figure 11: Regional volume versus surface area per GA. 
icance $(p \leq 0.001)$ for all tests except test between GA15.5 and GA16.5 in normalized surface to volume ratio $(p>0.05)$. Fig. 13, depicts the bull's eye plots of the results of the statistical test between GAs for each region. In the first column, corresponding to the test results between GA14.5 and GA15.5 values, FD measurements suggest that the development concentrates in the septal wall, while S2R and SR2VR measurements suggest that the changes are located in the lateral wall. The GA15.5-16.5 column seems more or less consistent across measurements without any reliably identifiable differences. All the measurements, especially FD and SR2VR, agree that the largest change happens from GA16.5 to GA17.5 in the area of papillary muscles. The GA17.5-18.5 column is again consistent across measurements with no reliable differences.

The aforementioned results can be observed in the fig. 14. The figure depicts the visualizations of the original data with one dataset per GA. The first column depicts the whole datasets in the same scale with their septoinferior part removed to be able to see their detailed cardiac anatomy. Consecutive columns represent anterior, inferior, lateral and septal cut of the corresponding datasets.

Fig. 15 represents a scatter plot of FD versus the myocardial volume to surface area ratio for all 361 regions of the 5 GAs. The correlation coefficients with $95 \%$ confidence intervals between FD and the volume to surface area ratio for each GA are the following: GA14.5: $-0.633[-0.691,-0.567]$, GA15.5: $-0.275[-0.368,-0.177]$, GA16.5: 0.082 [0.022, 0.183], GA17.5: $-0.353[-0.440,-0.259]$, GA18.5: $-0.489[-0.564,-0.407]$. From the plot we can observe that myocardial volume to surface area ratio separates data better than FD. If we look at the regional values of the FD for each GA separately we can observe that they all fall within the same range: $2-2.6$. On the other hand regional volume to surface area values occupy different ranges for different GAs, thus providing means to cluster data into regions corresponding to different GAs.

\section{Conclusions}

In this paper, we presented a framework for regional cardiac ventricular myocardial complexity analysis and visualization using a standardized representation of the LV to ensure inter-individual as well as longitudinal comparison. We also extended the current state of the art method for FD-based evaluation of trabecular complexity analysis to 3D, where we perform the 3D 


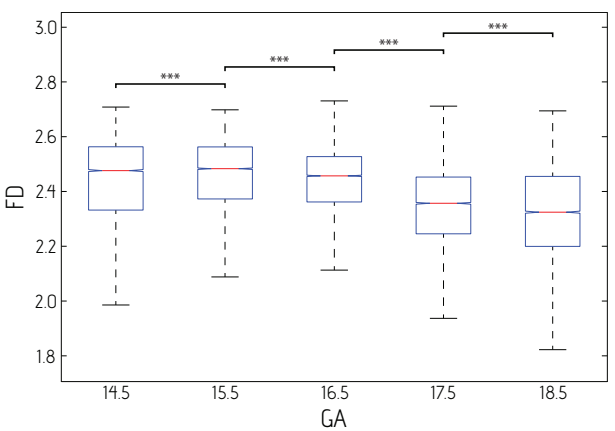

(a) FD

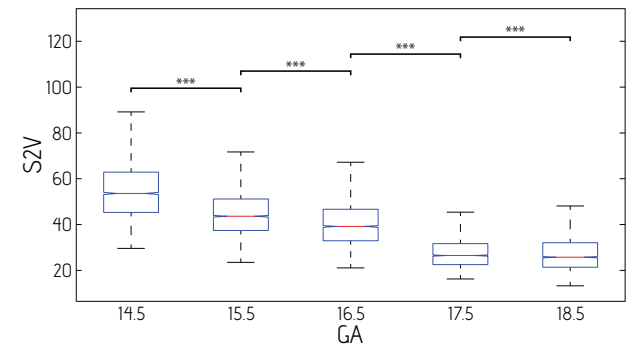

(b) S2V

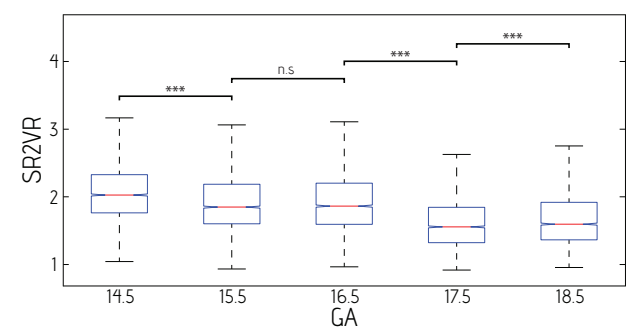

(c) SR2VR

Figure 12: Box plots of calculated (a) - fractal dimensions, (b) - surface-volume ratios and (c) - normalized surface-volume ratios for all regions of the data grouped by GA. For all calculated measures we performed two sided Wilcoxon rank sum test between GAs, where asterisks indicate significant differences between two groups. $* * *-p \leq 0.001$, n.s. - no significance $(p>0.05)$. In the plots the box represents the interquartile range (IQR), the edges of the box are the 25th and 75th percentiles, and the whiskers represent minimum and maximum values. 


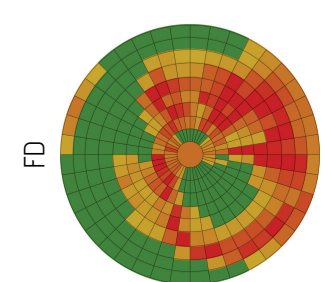

GA 14.5-15.5

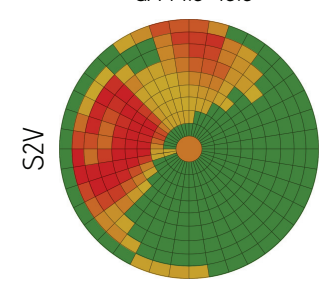

GA 14.5-15.5

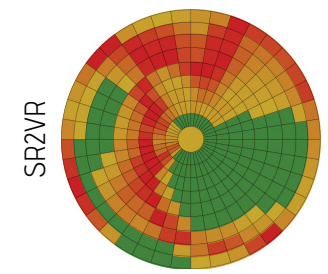

GA 14.5-15.5

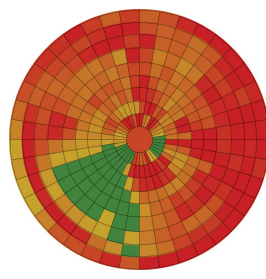

GA 15.5-16.5

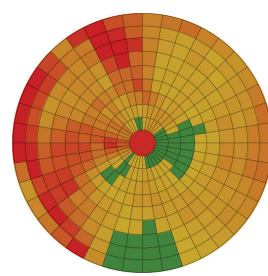

GA 15.5-16.5

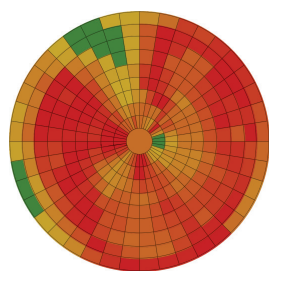

GA 15.5-16.5

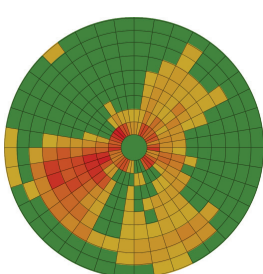

GA 16.5-17.5

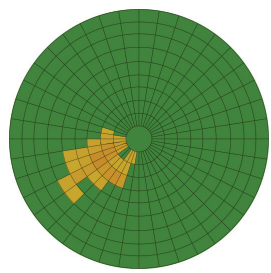

GA 16.5-17.5

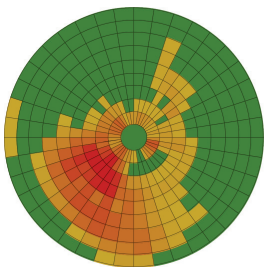

GA 16.5-17.5

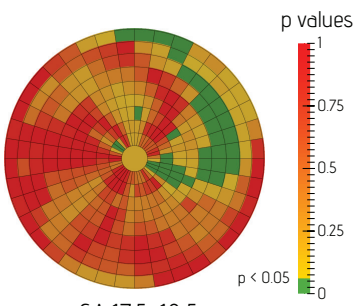

GA 17.5-18.5

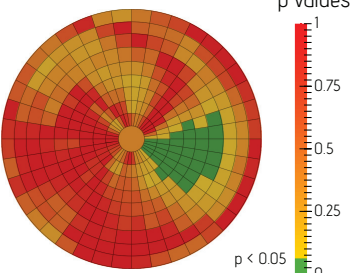

GA 17.5-18.5

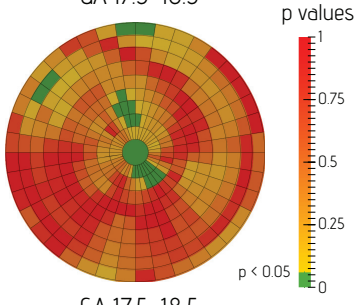

GA 17.5-18.5

Figure 13: Results of two sided Wilcoxon rank sum test between GAs for each region. Test performed for fractal dimension (FD), surface-volume ratio $(\mathrm{S} 2 \mathrm{~V})$ and normalized surface-volume ratio (SR2VR). 


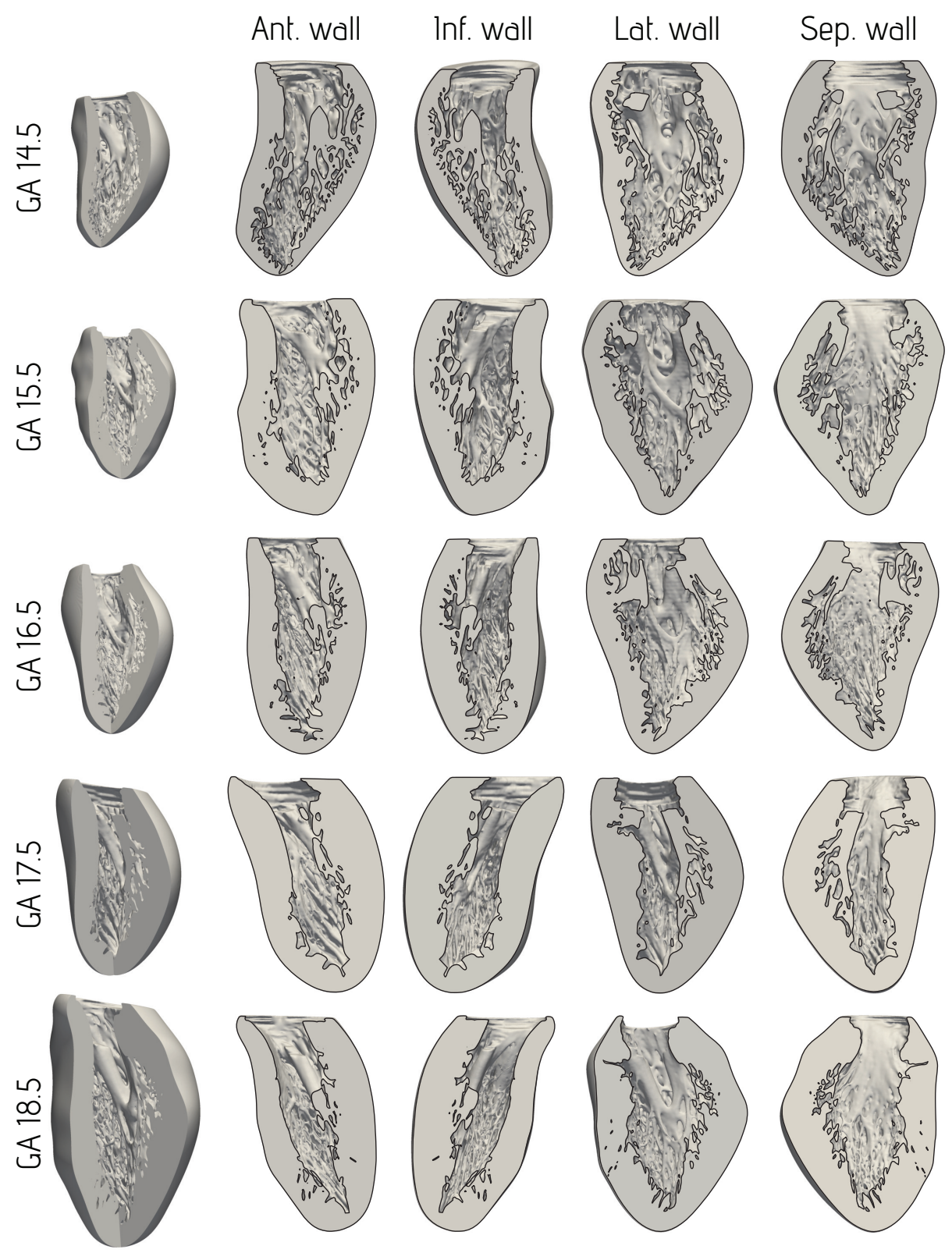

Figure 14: Visualization of one dataset from all gestational ages (GA). First column shows the whole datasets on the same scale with their septoinferior part removed to show their detailed cardiac anatomy. The other columns represent anterior, inferior, lateral and septal cut of the datasets. 


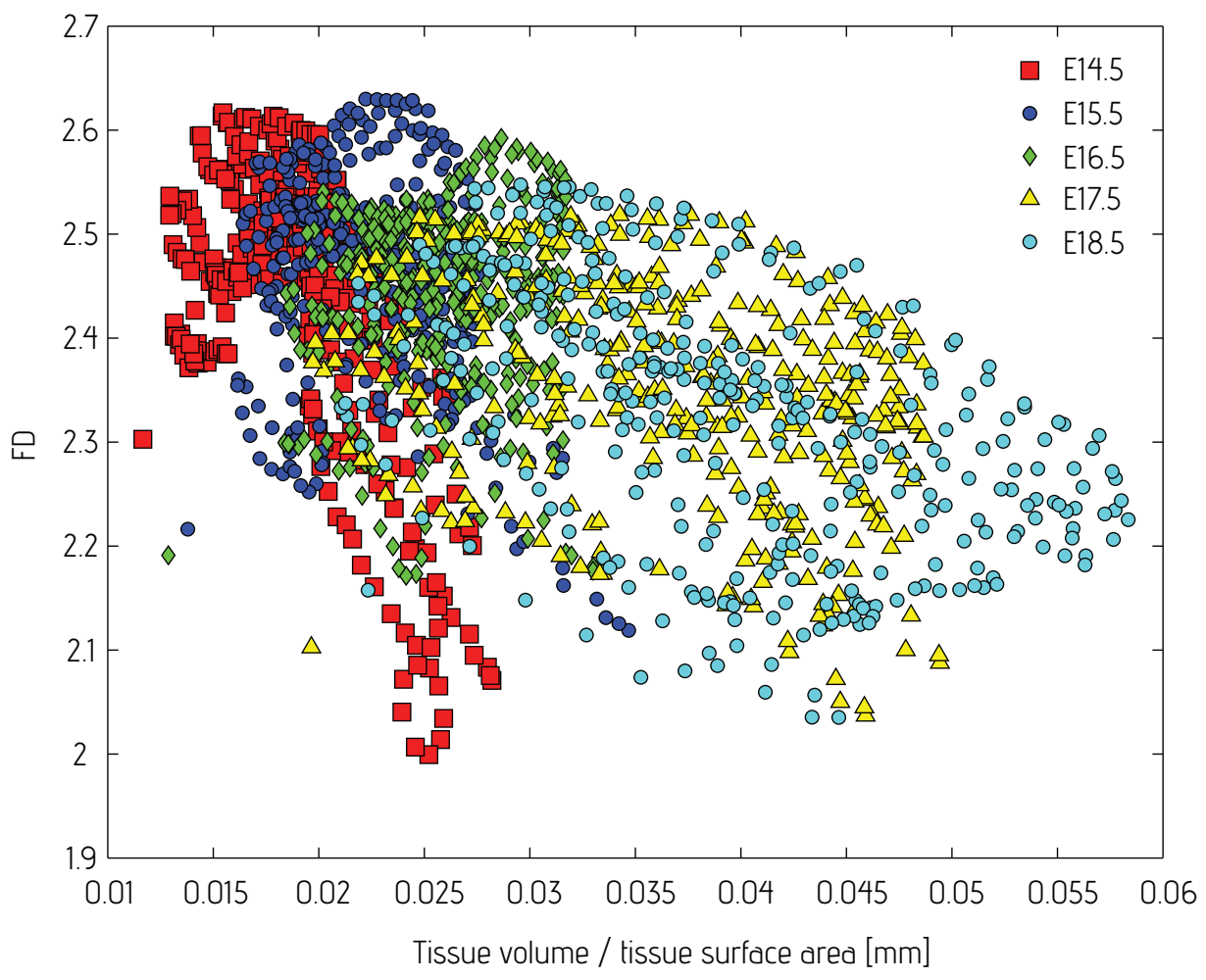

Figure 15: Regional FD versus volume-surface ratio per GA. 
fractal analysis locally on LV regions. The fractal analysis was extended with new proposed complexity measures such as surface ratios, occupied volume and surface-volume ratios. The obtained results are in agreement with the results obtained by Captur et al. (2016), where we get comparable results in trabecular complexity trends within the same gestational ages.

However, the Captur et al. method, performs 2D per-slice analysis and we have found no other reported results on regional trabecular complexity, defined either by the 17 American Heart Association (AHA) regions or any other variant. Therefore, to the best of our knowledge, this is the first paper to perform a 3D regional myocardial complexity analysis, both spatially and longitudinally. Our results confirm the known decrease in trabecular complexity with GA in normal (mouse) embryos, where the most complex regions at 18.5 embryonic day coincide with the regions where papillary muscles are established.

The proposed 3D fractal analysis, as compared to the $2 \mathrm{D}$, allows for a more accurate quantification of the 3D myocardial wall and the proposed analysis framework allows to apply previously inaccessible methods of quantification. The 3D FD still inherits some of the drawbacks of the 2D approach. When applied to natural fractals, FD calculation has a lack of consensus with respect to the choice of parameters, which most of the times are chosen using visual inspection and based on a trial and error basis and the computational cost. The proposed area and volume measures provide a more clearly defined and more interpretable complexity measure: the increase in myocardial volume is related to the growth of the compact myocardial layer or density increase, while surface area is simply the area of the interface between the blood and the tissue, directly proportional to the apparent complexity. The proposed approach allows for the quantification and comparison of normal, as well as abnormal, trabecular development and provides a way for the more objective quantification of alterations induced by genetic mutations.

Although results of our fractal analysis provided a similar finding as in Captur et al. (2016), in this study we provide a more comprehensive analysis of left ventricular myocardial complexity. We do a 3D extension as well as mapping to a reference, which allows us to study regional changes, in similar locations, in much more detail. The performed analysis might not reveal a lot of novel insight in normal heart development, as the study was performed on normal mice where the overall heart geometry is fairly constant, but offers novel possibilities to study abnormal development, as in for example congenital heart disease, wherewere using 2D slices would not easily provide 
comparable information in individuals with complex geometrical changes. Additionally, we provided previously unused complexity measures in cardiac development, related to volumes and areas, that could be more easily linked with for example future computational studies to investigate the relation between trabeculations, wall stress and perfusion by diffusion.

\section{Acknowledgments}

B. Paun is supported by the grant FI-DGR 2014 (2014 FI B01238) from the Generalitat de Catalunya. The research leading to these results has received funding from the EU FP7 for research, technological development and demonstration under grant agreement VP2HF (no. 611823) and from the Spanish Ministry of Economy and Competitiveness (grant TIN2011-28067, TIN2014-52923-R, the Maria de Maeztu Units of Excellence Programme MDM-2015-0502) and FEDER. C. Butakoff is supported by the grant from the Fundació La Marató de TV3 (№20154031), Spain. The HREM datasets used in this manuscript were provided and collected by Dr. T. J. Mohun, Emily Hardman and Fabrice Prin from the Francis Crick Institute, London.

\section{References}

Captur, G., Syrris, P., Obianyo, C., Limongelli, G., Moon, J.C., 2015. Formation and malformation of cardiac trabeculae: Biological basis, clinical significance, and special yield of magnetic resonance imaging in assessment. Canadian Journal of Cardiology 31, 1325-1337.

Captur, G., Wilson, R., Bennett, M.F., Luxán, G., Nasis, A., et al., 2016. Morphogenesis of myocardial trabeculae in the mouse embryo. Journal of anatomy $229,314-325$.

CIBC, 2015. Seg3D: Volumetric image segmentation and visualization. Scientific Computing and Imaging Institute (SCI), Download from: http://www.seg3d.org [online: 10/08/2015].

Dawson, D.K., Maceira, A.M., Raj, V.J., Graham, C., Pennell, D.J., et al., 2010. Regional thicknesses and thickening of compacted and trabeculated myocardial layers of the normal left ventricle studied by cardiovascular magnetic resonance. Circulation: Cardiovascular Imaging , CIRCIMAGING-110. 
Foroutan-Pour, K., Dutilleul, P., Smith, D., 1999. Advances in the implementation of the box-counting method of fractal dimension estimation. Applied mathematics and computation 105, 195-210.

Gati, S., Chandra, N., Bennett, R.L., Reed, M., Kervio, G., et al., 2013. Increased left ventricular trabeculation in highly trained athletes: do we need more stringent criteria for the diagnosis of left ventricular non-compaction in athletes? Heart 99, 401-408.

Goñi, J., Sporns, O., Cheng, H., Aznárez-Sanado, M., Wang, Y., et al., 2013. Robust estimation of fractal measures for characterizing the structural complexity of the human brain: Optimization and reproducibility. NeuroImage 83, 646-657.

Hentschel, H., Procaccia, I., 1983. The infinite number of generalized dimensions of fractals and strange attractors. Physica D: Nonlinear Phenomena $8,435-444$.

Jelinek, H.F., Jones, C.L., Warfel, M.D., Lucas, C., Depardieu, C., et al., 2006. Understanding fractal analysis? the case of fractal linguistics. Complexus 3, 66-73.

Karperien, A.L., Jelinek, H.F., 2016. Box-counting fractal analysis: A primer for the clinician, in: The Fractal Geometry of the Brain, pp. 13-43.

Kovacevic-Preradovic, T., Jenni, R., Oechslin, E., Noll, G., Seifert, B., et al., 2008. Isolated left ventricular noncompaction as a cause for heart failure and heart transplantation: A single center experience. Cardiology 112, 158-164.

Latour, L.L., Mitra, P.P., Kleinberg, R.L., Sotak, C.H., 1993. Timedependent diffusion coefficient of fluids in porous media as a probe of surface-to-volume ratio. Journal of Magnetic Resonance 101, 342-346.

Lorensen, W.E., Cline, H.E., 1987. Marching cubes: A high resolution 3d surface construction algorithm, in: ACM siggraph computer graphics, ACM. pp. 163-169.

Mohun, T.J., Weninger, W.J., 2012. Embedding embryos for high-resolution episcopic microscopy (HREM). Cold Spring Harbor Protocols 2012, pdbprot069583. 
Oechslin, E., Jenni, R., 2011. Left ventricular non-compaction revisited: a distinct phenotype with genetic heterogeneity? European heart journal $32,1446-56$.

Oechslin, E.N., Attenhofer Jost, C.H., Rojas, J.R., Kaufmann, P.A., Jenni, R., 2000. Long-term follow-up of 34 adults with isolated left ventricular noncompaction: A distinct cardiomyopathy with poor prognosis. Journal of the American College of Cardiology 36, 493-500.

Paun, B., Bijnens, B., Iles, T., Iaizzo, P.A., Butakoff, C., 2017. Patient independent representation of the detailed cardiac ventricular anatomy. Medical image analysis 35, 270-287.

Penela, D., Bijnens, B., Doltra, A., Silva, E., Castel, M.A., et al., 2013. Noncompaction cardiomyopathy is associated with mechanical dyssynchrony: A potential underlying mechanism for favorable response to cardiac resynchronization therapy. Journal of cardiac failure 19, 80-86.

Ritter, M., Oechslin, E., Sütsch, G., Attenhofer, C., Schneider, J., et al., 1997. Isolated noncompaction of the myocardium in adults, in: Mayo Clinic Proceedings, pp. 26-31.

Russell, D.A., Hanson, J.D., Ott, E., 1980. Dimension of strange attractors. Physical Review Letters 45, 1175-1178.

Sahoo, P., Wilkins, C., Yeager, J., 1997. Threshold selection using renyi's entropy. Pattern recognition 30, 71-84.

Schindelin, J., Arganda-Carreras, I., Frise, E., Kaynig, V., Longair, M., et al., 2012. Fiji: an open-source platform for biological-image analysis. Nature methods 9,676 .

Weninger, W.J., Geyer, S.H., Mohun, T.J., Rasskin-Gutman, D., Matsui, T., et al., 2006. High-resolution episcopic microscopy: A rapid technique for high detailed 3D analysis of gene activity in the context of tissue architecture and morphology. Anatomy and embryology 211, 213-221.

Yoo, T.S., Ackerman, M.J., Lorensen, W.E., Schroeder, W., Chalana, V., et al., 2002. Engineering and algorithm design for an image processing api: A technical report on itk-the insight toolkit. Studies in health technology and informatics , 586-592. 
Yuan, R., Nagao, T., Paré, P.D., Hogg, J.C., Sin, D.D., et al., 2010. Quantification of lung surface area using computed tomography. Respiratory research 11, 153.

Zhao, G., Denisova, K., Sehatpour, P., Long, J., Gui, W., et al., 2016. Fractal dimension analysis of subcortical gray matter structures in schizophrenia. PloS ONE 11, e0155415. 